\title{
Sobre cómo enfrentar el tema corrupción desde el arbitraje y los mecanismos existentes*
}

\author{
Danny Quiroga Anticona** \\ Recibido/Received: 15/06/2018 \\ Aceptado/Accepted: 16/07/2018
}

SuMARIO: 1. Introducción: El efecto de la corrupción y el arbitraje. 1.1 Acuerdos internacionales contra la corrupción: ¿son relevantes? 1.2 Experiencia internacional: algunos casos emblemáticos. 2. El accionar sua sponte del Tribunal. 2.1. Identificación de evidencias: la evidencia circunstancial. 2.1.1 El traslado de la carga de la prueba. 2.1.2 El estándar de la prueba: estándares mínimo-máximo. 2.1.3 El balance de probabilidades. 2.2 El entrenamiento del árbitro. 3. Doctrina clean hands. 4. Consecuencias de hallazgos comprobados de corrupción: el árbitro no es juez. 5. A manera de conclusión.

* El presente artículo es una adaptación hecha a un trabajo que el autor realizó previamente analizando el tema del tratamiento de la corrupción dentro del arbitraje peruano, de acuerdo al marco regulatorio local e internacional. Sin embargo, el aspecto de la práctica internacional resulta el relevante y pertinente para ser aplicado dentro de la práctica moderna arbitral internacional, coherente con los tiempos en que la creciente corrupción suele buscar nuevas formas para poder legitimar tales actos, cuestionables desde todo punto de vista. El artículo original salió publicado en la Revista de Arbitraje de la Pontificia Universidad Católica del Perú, <goo.gl/1sS3HQ>, y en la revista digital <www.enfoquederecho.com>.

** Abogado, titulado en la Universidad de San Martín de Porres, con estudios de maestría en Derecho Internacional Privado en la Universidad Johannes Gutenberg en Mainz, Alemania. Socio en arbitrajes del Estudio Paitan \& Abogados, especializado en temas de arbitraje internacional tanto en materia comercial como de inversiones. Asimismo, abogado en disputas relativas a las contrataciones del Estado. Correo electrónico: dquiroga@estudiopaitan.com

D. Quiroga, "Sobre cómo enfrentar el tema corrupción desde el arbitraje y los mecanismos existentes", Revista Ecuatoriana de Arbitraje, No. 9, 2017. 
Palabras Clave: corrupción, metodología, análisis.

KEYWORDS: corruption, methodology, analysis.

RESUMEN: El presente trabajo dedicado al tema corrupción busca explicar la metodología que hoy en día trata estos casos especiales y que no son más (o no deberían serlo) soslayados. Para ello, se busca dar a conocer tales avances a fin de saber cómo acercarse al tema y cómo tratarlo al interior de un proceso arbitral. Un aspecto que se analiza es la experiencia internacional: casos emblemáticos a partir de los cuales se ha desarrollado en parte la metodología y que en los tribunales arbitrales modernos resultan ser de práctica usual cuando existen casos que presenten tales elementos de corrupción. Otro aspecto bajo comentario es la explicación de los métodos de análisis en forma detallada y, finalmente, un tema importante, es la consecuencia de hallazgos sobre corrupción dentro de un proceso, el accionar del árbitro, el rol que toma al momento de emitir su decisión.

Aвstract: This article, which is dedicated to the topic of corruption, seeks to explain the methodology that is nowadays used for these special cases and that are no longer (or should not be) ignored. To do this, the article tries to make known such advances in order to know how to approach the issue and how to deal with it within an arbitration process. An aspect that is analyzed is the international experience: emblematic cases from which the methodology has been, in part, developed and, which in modern arbitration courts turn out to be of usual practice when there are cases that present such elements of corruption. Another aspect which is commented is the detailed explanation of the methods of analysis and, finally, an important issue is the consequence of findings about corruption within a process, the actions of the arbitrator and the role he takes issuing his decision. 


\section{INTRODUCCIÓN: EL EFECTO DE LA CORRUPCIÓN Y EL ARBITRAJE}

La corrupción adopta muchas formas y suele mezclarse muchas veces en actos que han sido en apariencia efectuados válidamente. Siendo esto así se puede manifestar en simples entregas de dinero sobornos, prebendas, fraudes, lavado de dinero y otros más, lo que tiene como consecuencia dificultar el poder detectar el momento del vicio. Adicionalmente debe manifestarse que la definición legal de corrupción en el mundo varía y es por ello que la forma en que será combatida la corrupción dependerá de la ley aplicable y la sede arbitral.

El arbitraje como jurisdicción reconocida en muchos países tiene sus propios niveles normativos a los que se acude para solucionar una controversia, así tenemos de manera muy genérica primero el convenio arbitral, las reglas de conducción del proceso, la ley aplicable y finalmente las prácticas internacionales. Dado que usualmente no existen disposiciones directas referidas a cómo afrontar un caso teñido de corrupción, es que un árbitro atento y experimentado deberá hacer uso de aquella(s) fuente(s) que le permita dentro del proceso que ventila poder reconocer, analizar y evaluar los elementos de corrupción en relación con la resolución de controversia planteada, que es justamente el encargo dado que le respalda la institución arbitral.

En el campo de las contrataciones del Estado, la corrupción ha estado presente muchas veces (sucede hoy día). Como ejemplo bastará el caso típico de aquella empresa privada que resulta ser la ganadora de la Buena Pro de un proceso de selección, habiéndose valido de actos de corrupción coludida con funcionarios públicos en la elaboración premeditada de los términos de referencia. Asimismo, la empresa que sin mayor experiencia conforma un consorcio, pero lo hace bajo control del funcionario público para que le sirva de canal formal en recibir dinero mal habido, la celebración de transacciones extrajudiciales con reconocimiento de daños y perjuicios al contratista con cláusulas ar- 
bitrales, entre otros. De ser detectados tales elementos por el árbitro, ¿debe mantenerse al margen de los mismos el árbitro o el tribunal?

En ese sentido, ¿qué posibilidades y bajo qué marco puede un árbitro actuar sobre todo cuando detecta que el caso que se encuentra ventilando contiene matices que develan vicios de corrupción subyacentes? El ámbito de acción del árbitro es un tema ampliamente estudiado, y resulta esencial la comprensión de este aspecto porque servirá de base para que en el análisis prospectivo que lleve a cabo el árbitro no soslaye elementos de corrupción. Si bien es cierto el campo de actuación arbitral está determinado por el acuerdo entre las partes (convenio arbitral); también es cierto que está determinado por las leyes que regulan la materia arbitral, como bien indica BorN ${ }^{1}$, la base de la función arbitral no puede ser alterada por el convenio arbitral, cuando existe un evidente perjuicio a una de las partes, es por ello que en esta función se deben observar las normas imperativas. Esta interacción de campos que determinan la capacidad decisoria arbitral resulta vital para poder pronunciarse en relación a elementos de corrupción y así mantener a salvo la jurisdicción arbitral de esos vicios.

Sin embargo, en la experiencia extranjera el tratamiento de la corrupción en materia arbitral tiene precedentes desde hace varias décadas, produciendo bastante doctrina y todo un tratamiento al respecto, contribuyendo de esta manera en fortalecer y capacitar más al árbitro con herramientas que le permitan no esquivar, sino incorporar el tema y además pronunciarse sobre el mismo ${ }^{2}$, lo que tendrá un efecto determinante en salvaguardar la institución arbitral. Se espera que esta contribución no sólo presente el tema, sino que además sirva para que sus mecanismos sean aplicados en aquellas controversias en arbitrajes que afronten planteamientos formales de incumplimientos contractuales

1. G. Born, International Commercial Arbitration, 1ra Ed., Kluwer, 2009, p. 1604.

2. Aunque en la actualidad es de observar que se vienen desarrollando foros internacionales en donde el tema principal es nuevamente la corrupción en los arbitrajes internacionales reflejando la preocupación de la comunidad internacional en retomar estos temas. El renombrado Institute of Transnational Arbitration (ITA, por sus siglas en inglés) desarrolló la importante conferencia ITA-ASIL sobre Corrupción en Arbitraje Internacional: Evidencia y Remedios el pasado abril de 2015 en Washington DC. 
que en el fondo contengan exigencias viciadas de prebendas, sobornos y otras modalidades ilícitas.

\subsection{Acuerdos internacionales contra la corrupción: ¿son relevantes?}

En todo proceso de análisis es importante conocer el entorno. Resulta pertinente dar una breve mirada al mundo y su posición frente a la corrupción. Desde hace varias décadas tanto diversos Estados, como organizaciones interestatales importantes han decidido no sólo efectuar declaraciones, sino además que las mismas sean instrumentos que estén a disposición de especialistas en aquellos casos que puedan estar viciados de corrupción. Esta postura denota una gran preocupación de la comunidad internacional en reducir, en lo posible, que la corrupción siga siendo soslayada y mucho menos que mine por dentro importantes mecanismos como el arbitraje.

Un primer pronunciamiento que abordó el asunto fue la Ley de Prácticas Corruptas en el Extranjero (American Foreign Corrupt Practices Act), la cual es una ley federal americana que desde 1977 prohíbe prácticas de corrupción en el extranjero por inversionistas americanos. A la fecha esta norma importante ha sido enmendada y cuenta desde el 2012 con una guía práctica para su aplicación. Es quizá el documento que motivó la generación de otros que fueron elaborados como convenios. Entre otros instrumentos destacables se encuentra: (i) la Convención para Combatir el Cohecho de Servidores Públicos Extranjeros en Transacciones Comerciales Internacionales ${ }^{3}$; (ii) la Convención de las Naciones Unidas contra la Corrupción ${ }^{4}$; y, (iii) la Ley Antisoborno de Reino Unido (UK Bribery Act) ${ }^{5}$.

3. Conocida como la OECD Anti Bribery Convention. Es una convención que nació a iniciativa de la Organización para la Cooperación y el Desarrollo Económico (OCDE) y desde 1997 ha servido de instrumento internacional para adecuar las normas internas.

4. Es un convenio multilateral en vigencia desde el 2003 y dispone medidas para evitar la corrupción doméstica e internacional. Perú es parte de este instrumento.

5. En el año 2010, el parlamento inglés expidió esta norma que, entre otras disposiciones, sanciona actos de corrupción en un amplio sentido. 
Además de estos documentos importantes de carácter general, es pertinente mencionar en lo relativo al mundo arbitral que una de las instituciones más prestigiosas en materia de arbitraje como es la Cámara de Comercio Internacional (en adelante, CCI) con sede en París, la cual en el año 2011 expidió la Cláusula ICC Anticorrupción ${ }^{6}$. KILCHES comenta al respecto que los diferentes puntos de vista de los tribunales arbitrales relativos al tema motivaron que la CCI expidiera la cláusula modelo anticorrupción, mediante la cual las partes acordaran expresamente que el tribunal estaría encargado en caso de problemas de corrupción ${ }^{7}$. Este es un avance, pero no la solución al tratamiento del problema.

Es por ello que consideramos que sí han sido y son relevantes todos estos instrumentos, primero porque están sirviendo de modelo para muchos Estados en reorientar sus sistemas jurídicos a fin de tomar una actitud firme contra la corrupción; segundo porque algunos de ellos en su aplicación son de orden internacional; $y$, tercero porque sirven para que el arbitraje adopte prácticas eficientes que permitan abordar profesionalmente estos asuntos. Si bien es cierto se está hablando de la experiencia internacional en el manejo del tema, considero que es perfectamente válida la adopción de estas prácticas en el arbitraje local de contrataciones públicas, más aún cuando la idea es evitar que la corrupción tan extendida encuentre un nicho de tolerancia en el arbitraje.

\subsection{Experiencia internacional: algunos casos emblemáticos}

Consideramos ilustrativo referirnos a algunos casos emblemáticos que se ocuparon de este asunto desde hace varias décadas para saber cómo ha sido la evolución y el tratamiento del mismo en la esfera arbitral.

6. Es un documento bastante interesante puesto que aborda el problema de manera frontal, cuenta con un glosario de prácticas prohibidas en su artículo 1 de la parte I, además de referirse a las políticas corporativas como medidas anticorrupción en su artículo 3 de la parte II.

7. R. KILCHES, "Die Behandlung des Einwandes von Korruption in Schiedsverfahren und bei der Anfechtung von Schiedssprüchen", SchiedsVZ, Vol. 3, Revista del Instituto Alemán de Arbitraje (DIS), 2016, p. 153. 
La primera referencia que se tiene es el emblemático caso arbitral llamado Lagergren Case ${ }^{8}$, el primer caso arbitral que trató el asunto de la corrupción. El caso en si estaba relacionado a un contrato mediante el cual una empresa británica le había encargado a un intermediario argentino, comisiones para obtener contratos de obras públicas en Argentina. El caso resultó ser particular porque el árbitro único, el profesor LAGERGREN, determinó que hubo actos de corrupción comprometidos con ese negocio. Esto motivó a que diera su célebre pronunciamiento mediante el cual denegó jurisdicción a las partes. En uno de los extremos de su pronunciamiento señalaría lo siguiente: "in the presence of a contract in dispute of the nature set out hereafter, condemned by public policy, decency and morality, I cannot in the interest of the administration of justice avoid examining the question of jurisdiction on my own motion" ${ }^{\prime \prime}$. El caso sigue siendo emblemático pues, si bien es cierto abordó el tema corrupción, fue criticado por la forma de resolver al declarar la no jurisdicción y no aplicar la doctrina de separabilidad del convenio arbitral para conservar su competencia, dejó abierto el camino para posteriores pronunciamientos por parte de tribunales arbitrales para afrontar el tema de fondo.

Un caso posterior es el que sucedió entre una compañía británica, la reclamante, y una compañía francesa ${ }^{10}$. La operación consistía en que la empresa británica brindaría servicios para proveer información y facilitar ganancias en la obtención de negocios con un gobierno africano. La operación en los documentos se presentaba como bastante seria, pero lo interesante está en que ciertas declaraciones de las partes y los testigos en el desarrollo del proceso, junto con otras evidencias, produjeron en el tribunal indicadores de que la operación en papeles distaba de lo que en realidad intentaban las partes ${ }^{11}$. Finalmente, el tribunal resolvió indicando que bajo la ley francesa el soborno es un ilícito e inmoral. Este caso, tal como lo expone MARTIN, se distanció del caso

8. CCI, Caso No. 1110, 1963.

9. E. Music, Corruption in International Commercial Arbitration: The Right of the Arbitrator to Conduct Self-initiated Investigation of Corruption, Tesis de Maestría en Derecho Procesal Internacional, Arbitraje Comercial Internacional. Universidad de Uppsala, 2013, p. 16.

10. CCI, Caso No. 3913, 1981.

11. T. MARTIN, "International Arbitration and Corruption: An Evolving Standard", International Oil, Gas \& Energy Dispute Management, Mineral Law Series Volume, 2002, p. 14. 
antes citado, puesto que el tribunal se declaró competente para arbitrar, declarando el consulting agreement nulo e inválido, vale decir, afrontó de una manera particular los méritos del caso.

Un ejemplo más. Una empresa demandante con sede en Liechtenstein y una empresa de construcción alemana ${ }^{12}$, ambas partes habían suscrito varios acuerdos básicos por un período de diez años en donde la demandante proveería de servicios en la búsqueda de contratos de construcción a la demandada en diversos países. La disputa tenía que ver con el pago de mayores porcentajes por una serie de acuerdos adicionales que tenían, sin embargo, al tribunal le llamó la atención uno de los acuerdos que disponía pagos, pero sin describir claramente los servicios a efectuar. Al ser este acuerdo algo inusual, el tribunal propuso que un experto independiente se encargue de revisar tales documentos y cuentas. Luego de una serie de objeciones, y luego de un largo análisis el tribunal determinó que el objeto real de ese acuerdo era el pago de sobornos. Este caso es muy ilustrativo puesto que demuestra cómo la evidencia documental, el discovery y las inferencias pueden determinar la suerte de las alegaciones de corrupción ${ }^{13}$. Además, en este caso el Tribunal desplazó la carga de la prueba (shifting the burden of proof) usualmente a cargo de quien alega los hechos y asumió la investigación.

Existen muchos casos arbitrales más que han ido trazando mejor el manejo del tema, estableciendo prácticas modernas efectivas relativas al tratamiento de controversias con evidentes elementos de corrupción camuflados. Son justamente estos casos los que nos servirán para poder tomar uno u otro mecanismo de evaluación a determinados elementos que no sean claros y que puedan tener incidencia con los resultados del proceso.

12. CCI, Caso No. 6497, 1994.

13. I. UluC, Corruption in International Arbitration, Tesis para la obtención del grado Doctor en Ciencias del Derecho (SJD). Universidad Estatal de Pensilvania, 2016, p. 216. 


\section{El ACCIONAR SUA SPONTE DEL TRIBUNAL}

El asunto central se encuentra ahora en cuál es la medida o acción que debe tomar un tribunal o un árbitro único en caso se halle frente a este tipo de procesos con serios visos de corrupción. La clave está contenida en el derecho/obligación sua sponte que tiene el tribunal para, en forma voluntaria, ingresar a hacer revisión de determinados aspectos que resulten dudosos en su obtención o finalidad. En si más que una medida es una actitud clara y proactiva que debe tener el tribunal, no tolerando elementos poco claros (corrupción alguna) en el proceso que está a su cargo.

FRIEDLAND, señala de manera bastante interesante que hay dos categorías de casos en los que los árbitros están justificados para hacer uso de su poder y sua sponte obtener evidencia. Un tipo de casos serían aquellos que implican asuntos de orden público, cuando consideran los árbitros han sido explorados inadecuadamente o no lo han sido, un ejemplo sería aquellos casos llamativos relativos a contratos que pueden haber sido obtenidos por corrupción, en este caso el uso del poder sua sponte no es controvertido; el otro tipo de casos, más interesante señala, son aquellos más complicados y menos llamativos en donde el asunto de si los árbitros deben ejercer su poder sua sponte para solicitar evidencia, en su opinión debe depender de la extensión a la cual el árbitro está obligado en la ordinaria operación de la carga de la prueba. No obstante, es claro en indicar que él considera que los árbitros sí tienen el poder ex officio para llamar a la producción de la prueba y en ambas categorías de $\operatorname{casos}^{14}$. Nuestra ley de arbitraje si concede esta herramienta a los árbitros, lo que veremos más adelante.

Esta herramienta es clave para que la actuación arbitral no quede inerme o pasiva ante hechos que se presenten de una forma tal que no se condiga con la realidad de la operación. Las modalidades de corrupción son bastas como hemos visto, por ello el hallazgo de la evidencia requerida será determinante para develar y resolver en forma correcta actos teñidos de corrupción.

14. P. FriedLAND, Fact-Finding by International Arbitrators - The Issue of Sua Sponte Calls for Evidence, ASA Conference, conferencia organizada por la Swiss Arbitration Association, 2015, p. 1. 
La doctrina hoy en día concuerda en el uso del poder de los árbitros para sua sponte solicitar evidencia; sin embargo, la crítica a esta práctica es referida a sus límites. ¿Cuál es el límite de un accionar sua sponte? Al respecto sostenemos que la motivación de buscar una evidencia está basada en una duda razonable, como sucediera en el famoso Caso No. 6497 de la $\mathrm{CCI}^{15}$, pues en este caso el tribunal halló que una de las adendas estaba referida a un pago exorbitante (no usual en esas prácticas comerciales) y no estaba plenamente justificado o identificado el motivo del mismo. Esto fue determinante para que el Tribunal ejerciera su poder sua sponte y entrara a solicitar más evidencias, comprobándose después que tal adenda del contrato era una que encubría pagos de sobornos. Sin embargo, consideramos que esta duda razonable de buscar mayores evidencias sua sponte en casos determinados, debe ser ejercida atendiendo: (i) el riesgo de emitir un laudo ultra petita; (ii) considerando los usos que conlleva en todo momento el debido proceso; y, (iii) no estableciendo sanciones. Estos, de alguna manera, podrían ser los límites que tendría que acatar todo árbitro frente a esta situación particular, lo contrario sería convalidar los actos que ocultan la corrupción.

En consecuencia, el accionar sua sponte de un Tribunal es completamente permitido, a partir de ello sostenemos que no sólo puede sino que debe ingresar a la búsqueda de la evidencia requerida. En ese sentido, como señala Mujic "la restricción del árbitro para decidir en materias no presentadas por las partes, queda en contraste con la obligación de emitir un laudo ejecutable. [....] [U]n árbitro, quien conlleva un rol jurisdiccional, no puede ignorar ilegalidades en orden de cumplir enteramente su rol, como árbitro de las partes"16. Teniendo claro lo que le está permitido al tribunal, la pregunta sería cómo se lleva a cabo en la realidad este accionar particular del tribunal. A continuación, algunas referencias.

15. CCI, N. 12. "With respect to product agreement Q, however, the arbitral tribunal found that there was a high enough degree of probability that the real object of product agreement Q was to channel bribes to officials in country X for this to be considered a case of bribery", en J. J. ARNALDEZ, Y. Derains y D. HASCHER, Collection of ICC Arbitral Awards 1996 - 2000, Kluwer Law, p. 233.

16. E. Mujic, N. 9, p. 41. 


\subsection{Identificación de evidencias: la evidencia circunstancial}

Hay que partir entendiendo que no existe un compendio procesal, ni nacional ni internacional al respecto; aquí la experiencia, el sentido común y el criterio de los árbitros van a jugar un rol importante. Entonces, estando ante una situación concreta en la cual los árbitros no dejarán pasar elementos que les hayan generado sospechas de corrupción, ingresamos a la fase de cómo el tribunal sua sponte debe conducirse a partir de estos hechos, para obtener una evidencia. Usualmente existen dos categorías de evidencias, la evidencia directa que vendría a ser esa prueba irrefutable y que determinará la suerte del caso y la otra categoría es la evidencia circunstancial, que es aquella detectada como consecuencia de la experiencia y la suspicacia ante los elementos presentados en el curso aparente normal de un proceso.

En casos con matices de corrupción, es muy raro hallar una evidencia directa que corrobore las sospechas. El hallazgo de una evidencia en si es una tarea compleja que se debe hacer en forma bastante aguda, a fin de evitar que la conducta que nos genera alerta, sea obviada y por el contrario sea contrastada con la evidencia requerida. Pero, ¿cuál es la evidencia requerida?

Señala SCHERER al respecto, en la práctica es muy raro que la prueba directa de la corrupción esté disponible, la mayoría de tribunales explica, han tenido que darse por satisfechos con la famosa prueba circunstancial ${ }^{17}$. La prueba circunstancial hallada deberá ser decisiva para poder establecer el nexo que nos lleve a determinar las sospechas de corrupción. En ese sentido, los casos que han abordado el tema en los últimos años han ido estableciendo ciertos procedimientos o estándares de obtención.

La regla general es que cada alegación de las partes deba estar acompañada de las pruebas que sustenten lo alegado. Pero en la práctica aquellos tribunales que han estado ligados en casos con elementos de corrupción han "tenido" que tomar ciertas dis-

17. M. Schrer, "Circumstantial Evidence in Corruption Cases before International Arbitral Tribunals". International Arbitration Law Review, Vol. 5, No. 2, 2002, p. 31. 
tancias de aquellas premisas básicas. Es ahí en donde algunos tribunales han hecho uso de la reversión de la carga de la prueba y/o reduciendo la condición del balance de probabilidades del estándar de la prueba ${ }^{18}$.

Un caso muy ilustrativo al respecto en temas de arbitraje de inversiones fue EDF Services v. Romania ${ }^{19}$, aquí se muestran aquellas dificultades encontradas por las partes intentando probar la corrupción en arbitraje internacional, en ese caso en el estado de las evidencias era improbable que el inversionista pueda tener éxito con su alegación de corrupción en el balance de probabilidades $^{20}$. Interesante ejemplo de cómo el proceso arbitral ha tenido que adecuarse a las circunstancias, en una suerte de simbiosis legal, a fin de no dejar elemento alguno que tenga como resultado un laudo que sea potencialmente anulable por ser contrario al orden público. En forma sucinta veremos en qué consisten estas técnicas para la búsqueda de evidencias.

\subsubsection{El traslado de la carga de la prueba}

En un proceso arbitral $u$ otro de cualquier naturaleza, es usual que quien plantee la demanda deba acompañar junto con su reclamo las pruebas pertinentes del hecho reclamado. KHVALEI señala que el traslado de la prueba es algo similar a hacer una presunción procesal ${ }^{21}$. La regla es simple en los casos donde una parte reclama que un contrato en cuestión encubre sobornos, el tribunal arbitral puede trasladar la carga de la prueba a la parte que reclama la legalidad de la transacción ${ }^{22}$. Esta es una de las formas de entender el traslado de la prueba que es una herramienta que va a permitir al Tribunal hacer un trabajo de prospección analítica y limpieza contra cualquier elemento ligado a la

18. M. HwANG y K. Lim, "Corruption in Arbitration — Law and Reality", Asian International Arbitration Journal, Número 1, 2012, p. 14.

19. ICSID, Caso No. ARB/05/13, 2008.

20. M. HWANG, y K. LiM, N. 18, p. 16.

21. V. KHVALEI, "Using red flags to prevent arbitration form becoming a safe harbor for contracts that disguise corruption", ICC International Court of Arbitration Bulletin, Vol. 24, Special Supplement, 2013, p. 24.

22. Ibídem. 
corrupción. No obstante, no es la única forma de hacer uso de la figura la carga de la prueba.

En un proceso arbitral ante la $\mathrm{CCI}^{23}$ el demandado argumentó que uno de los hechos base del reclamo del demandante había sido fraudulento. Esta era parte de la defensa que plantearon, sin embargo el Tribunal declaró que la parte que hacía esta alegación tenían que ser los primeros en producir un documento que generase un mínimo de confianza en la autenticidad de la alegación. Es decir, el Tribunal ante una seria acusación de la defensa de un acto de corrupción de inmediato consideró que ellos serían los primeros en tener que probar tal alegación ${ }^{24}$.

Sobre el traslado y reversión de la carga de la prueba se ha hablado y documentado bastante en los últimos años, de hecho en el mundo anglosajón es en donde esta práctica se ha desarrollado, casos en materia de construcción internacional tales como City Inn Ltd. v. Shepherd Construction Ltd. en Escocia en donde por primera vez se tocó el tema del traslado de la carga de la prueba ${ }^{25}$, Walker Construction Ltd. v. Quayside Homes Ltd. E Or, o Aspect Contract Ltd. v. Higgins Construction $\mathrm{Plc}^{26}$. Lo que ha hecho que esta práctica moderna del arbitraje permita ser una herramienta que los árbitros tengan a disposición ante situaciones que presentan algunas zonas grises en la controversia principal.

\subsubsection{El estándar de la prueba: estándares mínimo - máximo}

El estándar de la prueba no es otra cosa que el acercamiento que el tribunal va a tener frente a la prueba al momento de valorar la misma. El asunto es delicado puesto que obtenida la evidencia, que podría ser determinante para un pronunciamiento relativo a actos de corrupción, dependerá del tipo de valoración que realice el tribunal. Según GónZALEZ GARCía, hay diferentes enfoques res-

23. CCI, Award No. 10982, 2001.

24. A. Tweeddale y K. Tweeddale, Shifting the burden of proof: Revisiting Adjudication Decisions, $<$ goo.gl/G1o24D>, p. 5.

25. Ídem, p. 4.

26. Ídem, pp. 4-5. 
pecto al estándar de la prueba. Algunos sostienen que debe ser sobre la base de la preponderancia de las pruebas, otros al estándar denominado más allá de la duda razonable del sistema anglosajón penal, lo que implica un estándar alto de la prueba ${ }^{27}$.

Al respecto, LIEBSCHER ha realizado estudios interesantes sobre este asunto que no tiene una medida determinada, así él señala que hay dos diferentes estándares para aproximarse a la evidencia requerida para establecer corrupción. Una, la aproximación basada en la tradición del common-law que a su vez distingue entre una preponderance of evidence (estándar bajo) y un más alto estándar de clear and convincing evidence (estándar alto). La otra, señala el autor, en las jurisdicciones con tradición continental europea el estándar usado es si la evidencia disponible satisface el convencimiento del juez o el árbitro para la existencia de un hecho ${ }^{28}$.

Como vemos el tema es bastante subjetivo pues depende de varios factores como, grado de acusación de corrupción (de ser planteada por una de las partes), presunciones del propio tribunal en relación a operaciones ilegales de ser el caso, o vínculo entre el elemento de corrupción y el fondo de la discusión sobre la materia controvertida. Consideramos que sólo sopesando estos elementos en un proceso en particular se podría determinar el uso de determinado estándar de la prueba. Sin embargo creemos que es importante tener en cuenta que un alto grado probatorio en materia de corrupción hará difícil su probanza lo que implicará que la sospecha o denuncia sea finalmente no considerada a la hora de resolver, aún cuando esta exista en forma subyacente.

\subsubsection{El balance de probabilidades}

Este es un concepto afín al derecho anglosajón. Es la forma usual de valorar las pruebas, así tenemos que, entre los dos ex-

27. L. A. GonZÁLez GARCíA, "Corrupción, lavado de dinero y el arbitraje internacional”. Revista Latinoamericana de Derecho Comercial Internacional, Vol. 1, No. 1, 2013, p. 267.

28. C. Liebscher y F. Haugeneder, "Corruption and Investment Arbitration: Substantive Standards and Proof”, en C. Klausegger et al. (Ed.), Austrian Yearbook on International Arbitration, 2009, p. 545. 
tremos de valorar las pruebas de la tradición anglosajona antes comentada, opera un balance de probabilidades, el cual, en asuntos que tienen que ver con materias civiles pero con alegaciones criminales (corrupción), tiene como premisa "basta que la certeza sea superior al cincuenta por ciento de las posibilidades". Es decir, se parte de la idea que ambas partes en sus alegaciones y pruebas tienen la misma valoración frente al tribunal (cincuenta por ciento a cada uno), pero quien logre marcar la diferencia, podría determinar que en el balance de las probabilidades su alegación sea concluyente para el tribunal. Esta valoración también será de aplicación cuando un tribunal sua sponte halle evidencia de corrupción que incida con la materia en discusión y podría aplicar este método para poder sopesar las probabilidades.

El profesor Born trató este tema bastante y fue quien hizo famosa la frase que un balance de probabilidades debe ser asumido como "algo que es más probable, a que no lo sea"29.

A manera de ilustración, en el caso UNCITRAL EDF Services, el tribunal requirió pruebas claras y convincentes para la comprobación de alegaciones relativas a corrupción por parte del gobierno rumano. Luego de las audiencias y concluido el proceso el tribunal declaró respecto de tales alegaciones que el actor no cumplió con el estándar de prueba aplicable. El tribunal desestimó una serie de pruebas aportadas como documentos, correos, grabaciones, además declaró que el hecho había sido investigado ya por autoridades rumanas. Este tribunal ha sido objeto de crítica por parte de muchos tratadistas puesto que exigió un estándar probatorio demasiado alto, dejando de lado el estándar del balance de las probabilidades ${ }^{30}$, sobre todo respecto de hechos en donde la premisa more likley, than not resultaba aplicable.

Somos de la opinión que la determinación de la valoración de la prueba es decisión del árbitro o tribunal en base a los hechos ventilados. En casos excepcionales el pedido de pruebas de

29. G. BORN, N. 1, p. 1857.

30. J. TiRAdo y A. García, "Las Investigaciones de Corrupción por Autoridades Gubernamentales y el Arbitraje de Inversión: Los Desafíos Creados por la Creciente Convergencia de Dos Mundos Hasta Ahora (casi) Separados", Revista del Club Español de Arbitraje, No. 21, 2014, pp. 75-76. 
mayor exigencia puede resultar justificado, pero dificulta la toma de decisión sobre elementos de corrupción que puedan atentar contra el orden público. En ese sentido coincidimos con lo que los expertos KWAM y LIM sostienen que el balance de probabilidades debe ser aplicado atendiendo las tonalidades de cada caso, y no puede estar divorciado de las particularidades del mismo. Por ello es importante considerar la seriedad de las alegaciones ${ }^{31}$.

\subsection{El entrenamiento del árbitro}

¿Cómo debe prepararse entonces el árbitro que se encuentre ante tan compleja situación? Aquí es donde se requiere que el entrenamiento del árbitro, además de tener muy presente evaluar la calidad del árbitro a designar por las partes, aunque este no sea tema del presente artículo.

Este entrenamiento del árbitro no se obtendrá de otra manera sino que teniendo únicamente en cuenta lo siguiente: (i) sabiendo de los alcances del convenio arbitral; (ii) estando bastante informado del marco legal bajo el que actuará; (iii) adoptando las mejores prácticas arbitrales que permitan desarrollar su labor arbitral; (iv) evitando que su proceder entorpezca el proceso innecesariamente; $y$, sobre todo, en lo relativo al arbitraje en contrataciones públicas, $(\mathbf{v})$ conociendo muy bien su realidad y las prácticas dolosas que se han ido dando, a fin de determinar actos, conductas y demás elementos que se presenten en la consecución de contratos que muchas veces sirven de medio o son la finalidad de la corrupción.

En ese sentido, la idea central es el deber de un árbitro de emitir un laudo que no sólo esté comprometido con resolver una controversia de la mejor manera posible, sino teniendo en cuenta aspectos subyacentes que, de no ser contemplados, podrían acarrear que el laudo final legitime actos relacionados con la corrupción y sea posible de una anulación judicial. Es decir, el compromiso del árbitro con conducir un proceso y mantenerlo 31. M. Hwang y K. Lim, N. 18, p. 20. 
limpio es necesario, evitando caer en una pasividad que convalide actos de corrupción.

Para ello, es pertinente que en la práctica el árbitro se entrene: (i) valorando declaraciones de testigos; (ii) adoptando técnicas modernas de interrogación; (iii) identificando cláusulas oscuras de los contratos y documentos presentados con incidencia directa en la controversia planteada; (iv) sopesando actitudes de las partes en el proceso; (v) analizando la producción de documentos tanto la entrega como la denegatoria-; (vi) conjeturando posibles inferencias; y, (vii) entendiendo el modelo de negocio y prácticas usuales que no son empleadas en la industria materia de la controversia, y sus límites.

A fin de coadyuvar con esto y debido a la preocupación que genera el tema en el mundo arbitral actual, la Cámara de Comercio Internacional (CCI) ha elaborado una guía ${ }^{32}$ que resulta ser una ayuda de importante relevancia para los árbitros el día de hoy, y son las denominadas red flags. KHVALEI sostiene que las red flags pueden servir como una herramienta útil en el arbitraje para verificar si hay un riesgo de corrupción ${ }^{33}$.

En específico la guía del CCI antes indicada está básicamente dirigida a poner en sobre aviso y evitar actitudes destinadas a configurar pagos ilegales (sobornos) que se puedan dar en el desarrollo de los negocios. Son una suerte de listado de acciones o conductas que alertan de un eventual caso que podría estar relacionado con corrupción ${ }^{34}$. A pesar que las red flags en sí no pueden constituir violaciones a normas anti corrupción, ellas dan señales de advertencia que éstas deben ser tomadas en serio e investigadas $^{35}$. No vamos a reproducir cada una de estas alertas, pero sí resulta interesante por lo menos mencionar algunas, porque con-

32. Comisión en Responsabilidad Corporativa y Anti Corrupción de la Cámara de Comercio Internacional, ICC Guidelines on Agents, Intermediaries and Other Third Parties, $<\mathrm{goo} . \mathrm{gl} / \mathrm{hMYHFo}>$.

33. V. KhVAlei, N. 21, p. 23.

34. Véase, International Anti-Corruption Resource Center (IACRC), Guide to Combating Corruption \& Fraud in Development Projects, $<\mathrm{http}$ //guide.iacrc.org/the-red-flags-of-corruption-bid-rigging-collusive-bidding-and-fraud/> (11/08/2016).

35. Comisión en Responsabilidad Corporativa y Anti Corrupción de la Cámara de Comercio Internacional, N. 32, p. 5 . 
sideramos que muchas de ellas pueden ser de aplicación en nuestra realidad. En el listado aparece ya como señal de alerta a tener en cuenta:

- Aquellas operaciones que han tomado lugar en países conocidos por pagos de corrupción.

- Un tercero es sugerido por un funcionario público, particularmente uno con autoridad discrecional sobre el negocio del caso.

- Los pagos a servicios de terceros son desproporcionados en relación al servicio prestado.

Estas y otras conductas no son ajenas al proceder lamentable de muchos funcionarios y contratistas en el Perú que, como hemos leído antes, incluso actualmente están siendo procesados por la justicia. Por ello, es interesante leer y conocer las denominadas red flags que la CCI propone. Es pertinente, además, indicar que resultaría imposible que una guía contemple todas las eventuales señales de alerta no obstante ello es ya una iniciativa interesante y sobre todo una ayuda en el entrenamiento de los árbitros de observar diversos detalles que antes eran obviados.

\section{DOCTRINA CLEAN HANDS}

Es pertinente referirnos brevemente a la importancia de esta doctrina, la cual está contenida en un principio que cada vez está teniendo mayor resonancia en el mundo arbitral internacional; esto porque se busca que los actores de un proceso arbitral que sustenten sus reclamaciones estén libres de tintes de corrupción. El origen del principio proviene también del derecho anglosajón $\mathrm{y}$, en sencillo, significa que ninguna persona puede buscar una solución justa o sostener una defensa justa, si esa parte ha violado principios de justicia tales como la buena $\mathrm{fe}^{36}$. 
La doctrina en cuestión tiene un uso práctico y difundido puesto que actúa como catalizador dentro de un proceso, sea de la naturaleza que sea, a fin que los tribunales que tengan ante si partes con tales conductas manifiestas (contrarias a las normas), no sean acogidas dentro de su reclamación principal. Un tribunal de esta manera evita ser cómplice de eventuales legitimaciones de pretensiones basadas en corruptelas.

Este principio persigue mantener la integridad y equilibrio en un proceso, sin admisión alguna de vicios en el mismo, entendemos que es totalizante y determinante en la toma de decisiones. Con el acopio de información en el proceso, el análisis de las pruebas y la valoración de los demás elementos que hemos analizado, de llegarse a la conclusión que la(s) parte(s) habría(n) actuado contra las normas, se llegaría a la determinación que este principio sería vulnerado de no ser acogido, lo que tendría como consecuencia el enturbiamiento de un proceso que conllevaría al árbitro a pronunciarse desestimando la pretensión de fondo o declarando la no arbitrabilidad de la controversia por afectación del orden público.

En este punto consideramos que la invocación de este principio, rector de un proceso, servirá como base, únicamente ante pruebas que acrediten actuación de las partes contrarias a las normas, de desestimar la pretensión de fondo. Somos de la opinión que es importante tener presente que la actuación de las partes, que constituyan violación de las normas, debe tener una incidencia directa con la controversia planteada. Esto, sobre todo, puesto que el árbitro tiene una función doble, de un lado cumplir con el encargo contenido en el convenio arbitral, de otro lado evitar la convalidación de actos que sean contrarios al orden público.

Es importante que estas prácticas modernas sean cada vez más usuales y de mayor incidencia en los procesos arbitrales. Un proceso arbitral se nutre, consideramos, no sólo de las normas a las cuales está sometido el proceso, sino además de los principios internacionales del derecho y de las prácticas que estos principios han desarrollado, por ello su invocación en un 
proceso en donde esté plenamente acreditada la corrupción, no debería ser visto como un recurso exótico o sofisticado, sino como algo acorde a las tendencias que buscan fortalecer sistemas como el arbitral.

\section{Consecuencias de hallazgos comprobados de CORRUPCIÓN: EL ÁRBITRO NO ES JUEZ}

Aquí llegamos a la parte decisiva en la actuación del tribunal frente a tales hechos. ¿Cómo debe enfrentar el tribunal estos elementos que, luego de haber contrastado tales evidencias, ha llegado al pleno convencimiento que en el proceso existen elementos de corrupción? Como señala GonZÁLEZ GARCíA, son varias las opciones que se pueden adoptar al resolver un caso. Por un lado, el tribunal puede optar por no hacer una determinación sobre los alegatos de corrupción. Segundo, un tribunal podrá decidir que la demanda de arbitraje es inadmisible por considerar que la transacción violenta el orden público internacional. Por último, el tribunal podrá concluir que el contrato objeto del arbitraje es nulo o que la transacción es tipificada como una actividad ilegal según las normas obligatorias ${ }^{37}$.

En el caso peruano, el árbitro está en total capacidad conforme a la ley de arbitraje, según lo veremos, en pronunciarse, y en lo absoluto dejarlos de lado como parte de su decisión final, en salvaguarda de la institución arbitral. Asimismo, bajo la aplicación del principio kompetenz-kompetenz y la separabilidad del convenio arbitral, el tribunal se encuentra premunido de los elementos que le permitan declarar la jurisdicción sobre el caso y hacer el pronunciamiento que corresponda, y finalmente optar por una decisión definitiva dependerá de cada caso en particular y el impacto que hayan tenido estos elementos de corrupción en el contrato materia de controversia, de ahí que muchos consideren entre las diferentes variables: (i) ser inadmisible la demanda; 
(ii) ser admisible pero no arbitrable ${ }^{38}$, (iii) optar por la declaración de invalidez y nulidad del contrato.

Sobre la arbitrabilidad, el especialista ULUC realiza un comentario muy interesante, la doctrina de la arbitrabilidad reposa sobre una simple pregunta: ¿qué tipo de disputa puede ser sometida a arbitraje? Las partes podrían someter cualquier disputa, pero las leyes nacionales restringen esto. Por ejemplo las leyes nacionales mandatorias serían un factor determinante de la arbitrabilidad de la disputa ${ }^{39}$. Consecuentemente entendemos que disputas basadas en hechos que violaron las normas mandatorias, serían materias no arbitrables.

Sobre una declaración de nulidad e invalidez del contrato, en opinión de MuJIC, los árbitros son algo renuentes al considerar que el contrato entre las partes es el resultado de la autonomía de las partes. Esta renuencia de los árbitros es criticada por MujIC e indica que tal renuencia debe ser considerada como injustificada toda vez que la nulidad del contrato no va a invalidar el convenio arbitral y por ello el árbitro no pierde jurisdicción para pronunciarse en casos en que se presenten tales elementos ${ }^{40}$.

En opinión de ULUC, en el contexto de sobornos y otras prácticas corruptas, un contrato que promueve la corrupción o esté teñido de corrupción será contrario a las normas de orden público y no es previsto que sea ejecutable debido a lo inapropiado de la violación a las normas y costumbres y el antagonismo a la ley moderna $^{41}$. Entendemos, entonces, que atendiendo estas consideraciones se estaría ante la posibilidad de poder declarar en forma suficiente la nulidad e invalidez del contrato. PAVIC es de esta posición y es muy firme al señalar que no importa cuáles sean las reglas a las que un tribunal ha recurrido (nacionales o transnacionales), cuando examinando el potencial soborno, una vez este

38. La doctrina de la arbitrabilidad es compleja, pero en síntesis se considera que la declaración de arbitrabilidad está ligada a elementos que puedan ser transgresores de normas mandatorias o del orden público internacional y producto de ello, no ser arbitrables.

39. I. UluC, N. 13, p. 96.

40. E. MuJic, N. 9, p. 38.

41. I. Uluc, N. 13, p. 22. 
es hallado que bajo las leyes aplicables el soborno tomó lugar, el referido contrato que apuntaba al soborno, será declarado nulo e inválido. Debe ser resaltado que algunas veces esta consecuencia seguirá aún si la ley aplicable no trata una conducta particular como el soborno, ya que la sanción de nulidad puede devenir por el objeto de un contrato el cual es inmoral y consecuentemente ilegal $^{42}$.

\section{A MANERA DE CONCLUSIÓN}

El análisis del tema contenido en el presente artículo es complejo; no obstante, somos de la opinión que un asunto ligado al manejo arbitral en casos de corrupción, resulta ser de vital importancia. La preparación y entendimiento del tema por parte del árbitro es esencial, puesto que coadyuvará a que el arbitraje no sirva de subterfugio a este problema.

Es por ello que el fortalecimiento del sistema arbitral es una tarea de todos, académicos, expertos, abogados; pero sobre todo creemos que el trabajo es de quienes ejercen el poder decisorio en una contienda como son los árbitros, quienes deben asumir su rol sin temor alguno de ejercer las facultades que el convenio arbitral, la ley y conjuntamente con las buenas prácticas procesales le permiten.

Cada persona ligada al tema podrá obtener sus propias conclusiones, sin embargo queremos resaltar a manera de resumen los siguientes aspectos que a nuestro parecer son decisivos a tener en cuenta cuando se esté ante tal tipo de controversias:

- Es innegable la realidad que existen controversias derivadas de contratos gestados bajo actos de corrupción en el arbitraje de contrataciones públicas, por ello dejar de lado elemento o atisbo alguno de corrupción, implicaría que el arbitraje pierda vigencia por una falta de reacción en defender la pro-

42. V. PAVIC, "Bribery and International Commercial Arbitration. The Role of Mandatory Rules and Public Policy”, Victoria University of Wellington Law Review, Vol. 43, No. 4, 2012, p. 679. 
pia institución y sirva de refugio, siendo usado para lavar el provecho económico de hechos delictivos, perdiendo toda la credibilidad.

- El árbitro que tenga claro lo antes señalado deberá establecer el método de valoración, pero sopesando siempre el grado de implicancia del elemento de corrupción con relación a la controversia planteada. Es importante que el análisis evalué previamente qué dispone la Ley de Arbitraje local, a fin de poder ingresar a ver este tipo a fin que el árbitro o tribunal esté facultado para evaluar aspectos sua sponte que hayan surgido en el desarrollo del proceso.

- Finalmente es necesario que el árbitro (y las partes) entienda que él no es un Juez penal, que no se le pide que establezca una sanción o una pena privativa de la libertad a una persona en particular o entienda que con su decisión estaría vulnerando el principio de inocencia, tan sólo constatar que ante hechos que demuestren actos de corrupción subyacentes, afecta la materia que es puesta a resolución arbitral. 